\title{
Periodic Orbits of Radially Symmetric Keplerian-Like Systems with a Singularity
}

\author{
Shengjun $\mathrm{Li}^{1,2}$ and Yuming $\mathrm{Zhu}^{3}$ \\ ${ }^{1}$ School of Mathematics and Statistics, Central South University, Changsha, Hunan 410083, China \\ ${ }^{2}$ College of Information Sciences and Technology, Hainan University, Haikou 570228, China \\ ${ }^{3}$ Department of Mathematics and Physics, Qiongtai Teachers College, Haikou 570228, China
}

Correspondence should be addressed to Shengjun Li; shjli626@126.com

Received 22 December 2015; Accepted 15 March 2016

Academic Editor: Leszek Olszowy

Copyright (C) $2016 \mathrm{~S}$. Li and Y. Zhu. This is an open access article distributed under the Creative Commons Attribution License, which permits unrestricted use, distribution, and reproduction in any medium, provided the original work is properly cited.

\begin{abstract}
We study planar radially symmetric Keplerian-like systems with repulsive singularities near the origin and with some semilinear growth near infinity. By the use of topological degree theory, we prove the existence of two distinct families of periodic orbits; one rotates around the origin with small angular momentum, and the other one rotates around the origin with both large angular momentum and large amplitude.
\end{abstract}

\section{Introduction}

In recent few years, Fonda and his coworkers have studied the periodic, subharmonic, and quasiperiodic orbits for the radially symmetric Keplerian-like system

$$
x^{\prime \prime}+f(t,|x|) \frac{x}{|x|}=0, \quad x \in \mathbb{R}^{2} \backslash\{0\},
$$

in a systematic way, where $f \in C((\mathbb{R} / T \mathbb{Z}) \times(0, \infty), \mathbb{R})$ may be singular at the origin. See [1-6]. As mentioned in [5], many phenomena of the nature obey laws of (1), such as the Newtonian equation for the motion of a particle subjected to the gravitational attraction of a sun which lies at the origin.

Setting $\rho(t)=|x(t)|$, in [1], Fonda and Toader proved the case of solutions with large angular moment.

Theorem 1. Assume the hypotheses,

$$
\begin{gathered}
\lim _{\rho \rightarrow+\infty} \rho^{3} f(t, \rho)=+\infty, \\
\lim _{\rho \rightarrow+\infty} \frac{f(t, \rho)}{\rho}=0,
\end{gathered}
$$

uniformly for almost every $t$. Then, there exists $k_{1} \geq 1$ such that, for every integer $k \geq k_{1}$, (1) has a periodic solution $x_{k}(t)$ with minimal period $k T$, which makes exactly one revolution around the origin in the period time $k T$. Moreover,

$$
\begin{gathered}
\lim _{k \rightarrow \infty}\left(\min \left|x_{k}\right|\right)=+\infty, \\
\lim _{k \rightarrow \infty} \frac{\min \left|x_{k}\right|}{\max \left|x_{k}\right|}=1 ;
\end{gathered}
$$

if $\mu_{k}$ denotes the angular momentum associated with $x_{k}(t)$, then $\lim _{k \rightarrow \infty} \mu_{k}=+\infty$.

And in [2], they proved the case of solutions with small angular moment.

Theorem 2. Let the following two assumptions hold.

$\left(A_{1}\right)$ There are an integer $M$ and two constants $\alpha, \beta$ for which

$$
\begin{aligned}
\left(\frac{M \pi}{T}\right)^{2} & <\alpha \leq \liminf _{\rho \rightarrow+\infty} \frac{f(t, \rho)}{\rho} \leq \limsup _{\rho \rightarrow+\infty} \frac{f(t, \rho)}{\rho} \leq \beta \\
& <\left(\frac{(M+1) \pi}{T}\right)^{2},
\end{aligned}
$$

uniformly for every $t \in[0, T]$. 
$\left(\mathrm{A}_{2}\right)$ There are a positive constant $\delta$ and a continuous function $f:(0, \delta] \rightarrow \mathbb{R}$ such that

$$
\begin{gathered}
f(t, \rho) \leq h(\rho) \\
\text { for every } t \in[0, T] \text { and every } \rho \in(0, \delta], \\
\lim _{\rho \rightarrow 0^{+}} h(\rho)=-\infty \\
\int_{0}^{\delta} h(\rho) d \rho=-\infty
\end{gathered}
$$

Then, there exists $k_{2} \geq 1$ such that, for every integer $k \geq$ $k_{2}$, (1) has a periodic solution $x_{k}(t)$ with a minimal period $k T$, which makes exactly one revolution around the origin in the period time $k T$. Moreover, there is a constant $R>0$ such that, for every $k \geq k_{2}$,

$$
\frac{1}{R}<\left|x_{k}(t)\right|<R, \quad \text { for every } t \in \mathbb{R},
$$

and if $\mu_{k}$ denotes the angular momentum associated with $x_{k}(t)$ then $\lim _{k \rightarrow \infty} \mu_{k}=0$.

Following the notion in [7], we say that (1) has a repulsive singularity at the origin if

$$
\lim _{\rho \rightarrow 0^{+}} f(t, \rho)=-\infty, \quad \text { uniformly in } t \in \mathbb{R},
$$

whereas (1) has an attractive singularity at the origin if

$$
\lim _{\rho \rightarrow 0^{+}} f(t, \rho)=+\infty, \quad \text { uniformly in } t \in \mathbb{R} \text {. }
$$

Concerned with singular differential equations or singular dynamical systems, the question of the existence of periodic solutions is one of the central topics and therefore has attracted much attention [2, 3, 8-16]. More general systems, of the type

$$
x^{\prime \prime}+\nabla V(t, x)=0, \quad x \in \mathbb{R}^{n},
$$

were studied by many authors, mainly by use of variational methods; the singularities are of attractive type (see [1719]), where the potential $V(t, x)$ is $T$-periodic in $t$ and has singularities in $x$. When the singularities are of repulsive type, for the scalar singular equation

$$
x^{\prime \prime}+g(t, x)=0, \quad x>0
$$

we recall the following results. Let $g(t, x)=g(x)-e(t)$, where $g \in C\left(\mathbb{R}_{+}, \mathbb{R}\right)$ and $e \in C(\mathbb{R}, \mathbb{R})$ are $T$-periodic satisfying the following strong force condition at $x=0$ :

$$
\begin{array}{r}
\lim _{x \rightarrow 0^{+}} g(x)=-\infty, \\
\lim _{x \rightarrow 0^{+}} \int_{1}^{r} g(x) d x=+\infty,
\end{array}
$$

where $g$ is superlinear at $x=+\infty$ :

$$
\lim _{x \rightarrow+\infty} \frac{g(x)}{x}=+\infty
$$

Fonda et al. [20] used the Poincaré-Birkhoff theorem to obtain the existence of positive periodic solutions, including all subharmonics. Similarly, when $g(t, x)$ is superlinear at $x=+\infty$ and satisfies the strong force condition at $x=0$ that states that there are positive constants $c, c^{\prime}, v$ such that $v \geq 1$ and

$$
c x^{-v} \leq-g(t, x) \leq c^{\prime} x^{-v}
$$

for every $t$ and every $x$ sufficiently small, del Pino and Manásevich proved in [21] the existence of infinitely many periodic solutions to (10).

When $g(t, x)$ is semilinear at $x=+\infty$, del Pino et al. [22] proved the existence of at least one positive $T$-periodic solution of (10) if $g(t, x)$ satisfies (13) near $x=0$ and the following nonresonance conditions at $x=+\infty$ : there exists $k \in \mathbb{N}$ and a small constant $\varepsilon>0$ such that

$$
\left(\frac{k \pi}{T}\right)^{2}+\varepsilon \leq \frac{g(t, x)}{x} \leq\left(\frac{(k+1) \pi}{T}\right)^{2}-\varepsilon
$$

for all $t$ and all $x \gg 1$. The result was later improved by Yan and Zhang [23], conditions (14) are removed, and the existence of at least one positive solution under suitable nonresonance conditions is obtained by using the topological degree theory. We note that conditions (14) are the uniform nonresonance conditions with respect to the Dirichlet boundary condition, not with respect to the periodic boundary condition.

It seems that the periodic boundary value problem for singular differential equations is closely related to the Dirichlet boundary value problem. A relationship between periodic and Dirichlet boundary value problems for secondorder differential equations with singularities is established in [24]. Our main motivation is to obtain by $[1,2]$ that we will use such a relationship between the periodic boundary value problem and the Dirichlet boundary value problem to obtain the existence of two distinct families of periodic orbits to singular systems (1). Compared with Theorems 1 and 2, the main novelty in the paper is represented by the conditions at infinity, which remind us of a situation between the first and the second eigenvalue but are more general since the comparison involves the mean and the "weighted" eigenvalue associated with the functions controlling the ratio $f(t, \rho) / \rho$.

The main results in this paper are formulated in Theorem 6 and Theorem 17 (see them also for the precise statements). We summarize these two results informally.

Theorem 3. Let the following assumptions hold.

$\left(\mathrm{H}_{1}\right)$ There exist $v \geq 1$ and positive constants $c, c^{\prime}, \delta$, such that

$$
\frac{c}{\rho^{\nu}} \leq-f(t, \rho) \leq \frac{c^{\prime}}{\rho^{\nu}}
$$

for all t and all $0<\rho<\delta$.

$\left(\mathrm{H}_{2}\right)$ There exist T-periodic continuous functions $\psi, \Psi$ such that

$$
\psi(t) \leq \liminf _{\rho \rightarrow+\infty} \frac{f(t, \rho)}{\rho} \leq \limsup _{\rho \rightarrow+\infty} \frac{f(t, \rho)}{\rho} \leq \Psi(t)
$$


uniformly in t. Moreover,

$$
\begin{aligned}
\bar{\psi} & >0, \\
\underline{\lambda}_{1}(\Psi) & >0 ;
\end{aligned}
$$

here $\bar{\psi}=(1 / T) \int_{0}^{T} \psi(t) d t$ is the mean value, and $\underline{\lambda}_{1}(\Psi)$ is the $2 T$-periodic eigenvalues of

$$
\rho^{\prime \prime}+(\lambda+q(t)) \rho=0 .
$$

Then system (1) has two distinct families of periodic orbits with the following distinct behavior: one rotates around the origin with large angular momentum and large amplitude, and the other one rotates around the origin with small angular momentum.

The rest of this paper is organized as follows. In Section 2, some preliminary results will be given. In Section 3, by the use of topological degree theory, we establish the existence of periodic orbits with large momentum and large amplitude. In Section 4, the periodic orbits with small momentum are established.

\section{Preliminaries}

In this section, we present some results which will be applied in Sections 3 and 4. Let us first introduce some known results on eigenvalues. Let $q(t)$ be a $T$-periodic potential such that $q \in L^{1}(\mathbb{R})$. Consider the scalar eigenvalue problems of

$$
x^{\prime \prime}+(\lambda+q(t)) x=0
$$

with the periodic boundary condition,

$$
\begin{gathered}
x(0)=x(T), \\
x^{\prime}(0)=x^{\prime}(T),
\end{gathered}
$$

or with the antiperiodic boundary condition

$$
\begin{gathered}
x(0)=-x(T), \\
x^{\prime}(0)=-x^{\prime}(T) .
\end{gathered}
$$

We use $\lambda_{1}^{D}(q)<\lambda_{2}^{D}(q)<\cdots<\lambda_{n}^{D}(q)<\cdots$ to denote all eigenvalues of (19) with the Dirichlet boundary condition:

$$
x(0)=x(T)=0 .
$$

These eigenvalues, as a whole, are called the characteristic values of (19); the following are the standard results. See, for example, $[25,26]$.

$\left(E_{1}\right)$ With respect to the periodic and antiperiodic eigenvalues, there exist two sequences $\left\{\underline{\lambda}_{n}(q): n \in \mathbb{N}\right\}$ and $\left\{\bar{\lambda}_{n}(q): n \in \mathbb{Z}^{+}\right\}$such that

$$
\begin{aligned}
-\infty & <\bar{\lambda}_{0}(q)<\underline{\lambda}_{1}(q) \leq \bar{\lambda}_{1}(q)<\underline{\lambda}_{2}(q) \leq \bar{\lambda}_{2}(q) \\
& <\cdots<\underline{\lambda}_{n}(q) \leq \bar{\lambda}_{n}(q)<\cdots,
\end{aligned}
$$

where $\underline{\lambda}_{n}(q) \rightarrow+\infty, \bar{\lambda}_{n}(q) \rightarrow+\infty$ as $n \rightarrow+\infty$.

$\left(\mathrm{E}_{2}\right) \lambda$ is an eigenvalue of (19)-(20) if and only if $\lambda=\underline{\lambda}_{n}(q)$ or $\bar{\lambda}_{n}(q)$ with $n$ being even; and $\lambda$ is an eigenvalue of (19)-(21) if and only if $\lambda=\underline{\lambda}_{n}(q)$ or $\bar{\lambda}_{n}(q)$ with $n$ being odd.

$\left(E_{3}\right)$ The comparison results hold for all of these eigenvalues. If $q_{1} \leq q_{2}$ then

$$
\begin{aligned}
\underline{\lambda}_{n}\left(q_{1}\right) & \geq \underline{\lambda}_{n}\left(q_{2}\right), \bar{\lambda}_{n}\left(q_{1}\right) \geq \bar{\lambda}_{n}\left(q_{2}\right), \lambda_{n}^{D}\left(q_{1}\right) \\
& \geq \lambda_{n}^{D}\left(q_{2}\right)
\end{aligned}
$$

for any $n \geq 1$.

$\left(\mathrm{E}_{4}\right)$ The eigenvalues $\underline{\lambda}_{n}(q)$ and $\bar{\lambda}_{n}(q)$ can be recovered from the Dirichlet eigenvalues in the following way. For any $n \geq 1$,

$$
\begin{aligned}
& \underline{\lambda}_{n}(q)=\min \left\{\lambda_{n}^{D}\left(q_{t_{0}}\right): t_{0} \in \mathbb{R}\right\}, \\
& \bar{\lambda}_{n}(q)=\max \left\{\lambda_{n}^{D}\left(q_{t_{0}}\right): t_{0} \in \mathbb{R}\right\},
\end{aligned}
$$

where $q_{t_{0}}(t)$ denotes the translation of $q(t): q_{t_{0}}(t) \equiv$ $q\left(t+t_{0}\right)$.

$\left(\mathrm{E}_{5}\right) \bar{\lambda}_{n}(q), \underline{\lambda}_{n}(q)$, and $\lambda_{n}^{D}(q)$ are continuous in $q$ in the $L^{1}$ topology of $L^{1}(0, T)$.

$\left(\mathrm{E}_{6}\right) q(t)<(n \pi / T)^{2}$ a.e. $t \Rightarrow \lambda_{n}^{D}(q)>0$.

In order to prove our results, we need two preliminary results. The first one is the following global continuation principle of Leray-Schauder.

Lemma 4 (see [27, Theorem 14]). Let the operator $H$ : $\left[\mu_{1}, \mu_{2}\right] \times \bar{G} \rightarrow X$ be compact, where $G$ is a bounded open set in the Banach space $X$. Then equation

$$
x-H(\mu, x)=0, \quad \mu \in \mathbb{R}, x \in X,
$$

has a continuum $\mathscr{C}$ of solutions in $\mathbb{R} \times X$ which connects the set $\left\{\mu_{1}\right\} \times G$ with the set $\left\{\mu_{2}\right\} \times G$, if the following conditions are satisfied:

(I) $\operatorname{deg}(H, G) \neq 0$;

(II) (26) has no solution on $\left[\mu_{1}, \mu_{2}\right] \times \partial G$.

To state the second preliminary result, we recall some notation and terminology from [28]. Define $L$ : $\operatorname{dom} L \subset$ $X \rightarrow Z, L x=x^{\prime}$, a Fredholm mapping of index zero, with $\operatorname{dom} L=\{x \in X: x(\cdot)$ is absolutely continuous $\}$, where the Banach spaces $X, Z$ are given as

$$
\begin{aligned}
& X=\left\{x \in C\left([0, T], \mathbb{R}^{m}\right): x(0)=x(T)\right\}, \\
& Z=L^{1}\left([0, T], \mathbb{R}^{m}\right)
\end{aligned}
$$

with their usual norms. Let $M_{0}$ be the Nemitzky operator from $X$ to $Z$ induced by the map $f_{0}$; that is, $M_{0}: x(\cdot) \rightarrow$ $f_{0}(x(\cdot))$. Consider the equation

$$
L x=M_{0} x, \quad x \in \operatorname{dom} L .
$$


Lemma 5 (see [28, Theorem 1]). Let $\Omega \subset X$ be a bounded open subset and assume that there is no $x(\cdot) \in \partial_{X} \Omega$ such that $x^{\prime}=f_{0}(x)$. Then

$$
\operatorname{deg}_{L}\left(L-M_{0}, \Omega\right)=(-1)^{m} \operatorname{deg}_{B}\left(f_{0}, \Omega \cap R^{m}, 0\right),
$$

where $\operatorname{deg}_{L}, \operatorname{deg}_{B}$ denote the Schauder degree and the Brouwer degree, respectively.

We refer the reader to [29] for more details about degree theory.

\section{Periodic Solutions with a Large Angular Momentum}

We look for solutions $x(t) \in \mathbb{R}^{2}$ which never attain the singularity, in the sense that

$$
x(t) \neq 0, \quad \text { for every } t \in \mathbb{R} \text {. }
$$

Using the same idea in [1], we may write the solutions of (1) in polar coordinates

$$
x(t)=\rho(t)(\cos \varphi(t), \sin \varphi(t)) .
$$

Then we have the collisionless orbits if $\rho(t)>0$ for every $t$. Moreover, (1) is equivalent to the following system:

$$
\begin{array}{r}
\rho^{\prime \prime}+f(t, \rho)-\frac{\mu^{2}}{\rho^{3}}=0, \\
\rho^{2} \varphi^{\prime}=\mu,
\end{array}
$$

where $\mu$ is the angular momentum of $x(t)$. Recall that $\mu$ is constant in time along with any solution. In the following, when considering a solution of (32), we will always implicitly assume that $\mu \geq 0$ and $\rho>0$.

If $x(t)$ is $T$-radially periodic, then $\rho(t)$ must be $T$ periodic. We will look for solutions for which $\rho(t)$ is $T$ periodic. We thus consider the boundary value problem

$$
\begin{aligned}
\rho^{\prime \prime}+f(t, \rho) & =\frac{\mu^{2}}{\rho^{3}}, \\
\rho(0) & =\rho(T), \\
\rho^{\prime}(0) & =\rho^{\prime}(T) .
\end{aligned}
$$

Now we present our main result.

Theorem 6. Assume that $\left(H_{1}\right)$ and $\left(H_{2}\right)$ are satisfied. Then, (33) has a T-periodic solution, and there exists $K_{1} \geq 1$ such that, for every integer $k \geq K_{1}$, system (1) has a periodic solution $x_{k}(t)$ with minimal period $k T$, which makes exactly one revolution around the origin in the period time $k T$. Moreover,

$$
\begin{aligned}
\lim _{k \rightarrow \infty}\left(\min \left|x_{k}\right|\right) & =+\infty, \\
\lim _{k \rightarrow \infty} \mu_{k} & =+\infty .
\end{aligned}
$$

Now we begin by showing Theorem 6 and use topological degree theory. To this end, we deform the first equation of (33) to a simpler singular autonomous equation:

$$
\rho^{\prime \prime}+a \rho=\frac{1}{\rho},
$$

where $a$ for some positive constant satisfy $0<a<(\pi / T)^{2}$. Consider the following homotopy equation:

$$
\rho^{\prime \prime}+f(t, \rho ; \tau)-\frac{\mu^{2}}{\rho^{3}}=0, \quad \tau \in[0,1],
$$

where $f(t, \rho ; \tau)=\tau f(t, \rho)+(1-\tau)(a \rho-1 / \rho)$. We need to find a priori estimates for the possible positive $T$-periodic solutions of (36).

Note that $f(t, \rho ; \tau)$ satisfies the conditions $\left(\mathrm{H}_{1}\right)$ with the same $\nu$ and minor changes of $c, c^{\prime}, \delta$. Accordingly $f(t, \rho ; \tau)$ satisfies $\left(\mathrm{H}_{1}\right)$ uniformly with respect to $\tau \in[0,1]$. Moreover, for each $\tau \in[0,1], f(t, \rho ; \tau)$ satisfies (16) with $\psi=\psi_{\tau}=$ $\tau \psi(t)+(1-\tau) a$ and $\Psi=\Psi_{\tau}=\tau \Psi(t)+(1-\tau) a$. We will prove that $\psi_{\tau}$ and $\Psi_{\tau}$ satisfy (17) uniformly in $\tau \in[0,1]$. The usual $L^{p}$-norm is denoted by $\|\cdot\|_{p}$, and the supremum norm of $C[0, T]$ is denoted by $\|\cdot\|$. This follows from the convexity of the first eigenvalues with respect to potentials.

Lemma 7. Given $q_{0}, q_{1} \in L^{1}(0, T)$, then, for all $\tau \in[0,1]$,

$$
\begin{aligned}
& \lambda_{1}^{D}\left(\tau q_{1}+(1-\tau) q_{0}\right) \geq \tau \lambda_{1}^{D}\left(q_{1}\right)+(1-\tau) \lambda_{1}^{D}\left(q_{0}\right), \\
& \underline{\lambda}_{1}\left(\tau q_{1}+(1-\tau) q_{0}\right) \geq \tau \underline{\lambda}_{1}\left(q_{1}\right)+(1-\tau) \underline{\lambda}_{1}\left(q_{0}\right) .
\end{aligned}
$$

Proof. Put $q_{\tau}=\tau q_{1}+(1-\tau) q_{0}, \tau \in[0,1]$. Then

$$
\begin{aligned}
& \lambda_{1}^{D}\left(q_{\tau}\right)=\inf _{\substack{\varphi \in H_{0}^{1}(0, T) \\
\|\varphi\|_{2}=1}} \int_{0}^{T}\left(\varphi^{\prime 2}(t)-q_{\tau}(t) \varphi^{2}(t)\right) d t \\
& =\inf _{\substack{\varphi \in H_{0}^{1}(0, T) \\
\|\varphi\|_{2}=1}}\left(\tau \int_{0}^{T}\left(\varphi^{\prime 2}(t)-q_{1}(t) \varphi^{2}(t)\right) d t\right. \\
& \left.+(1-\tau) \int_{0}^{T}\left(\varphi^{\prime 2}(t)-q_{0}(t) \varphi^{2}(t)\right) d t\right) \\
& \geq \tau \inf _{\substack{\varphi \in H_{0}^{1}(0, T) \\
\|\varphi\|_{2}=1}} \int_{0}^{T}\left(\varphi^{\prime 2}(t)-q_{1}(t) \varphi^{2}(t)\right) d t+(1 \\
& -\tau) \inf _{\substack{\varphi \in H_{0}^{1}(0, T) \\
\|\varphi\|_{2}=1}} \int_{0}^{T}\left(\varphi^{\prime 2}(t)-q_{0}(t) \varphi^{2}(t)\right) d t \\
& =\tau \lambda_{1}^{D}\left(q_{1}\right)+(1-\tau) \lambda_{1}^{D}\left(q_{0}\right) .
\end{aligned}
$$

This proves (37). have

For (38), applying (37) to $q_{i}=q_{i, t_{0}}$, where $t_{0} \in[0, T]$, we

$$
\lambda_{1}^{D}\left(q_{\tau, t_{0}}\right) \geq \tau \lambda_{1}^{D}\left(q_{1, t_{0}}\right)+(1-\tau) \lambda_{1}^{D}\left(q_{0, t_{0}}\right)
$$


for all $t_{0}$. Thus

$$
\begin{aligned}
\lambda_{1}^{D}\left(q_{\tau}\right) & =\min _{t_{0}} \lambda_{1}^{D}\left(q_{\tau, t_{0}}\right) \\
& \geq \min _{t_{0}} \tau \lambda_{1}^{D}\left(q_{1, t_{0}}\right)+(1-\tau) \lambda_{1}^{D}\left(q_{0, t_{0}}\right) \\
& =\tau \min _{t_{0}} \lambda_{1}^{D}\left(q_{\tau, t_{0}}\right) \\
& \geq \min _{t_{0}} \tau \lambda_{1}^{D}\left(q_{1, t_{0}}\right)+(1-\tau) \min _{t_{0}} \lambda_{k}^{D}\left(q_{0, t_{0}}\right) \\
& =\tau \underline{\lambda}_{1}\left(q_{1}\right)+(1-\tau) \underline{\lambda}_{1}\left(q_{0}\right) .
\end{aligned}
$$

Hence (38) holds.

Note that

$$
\bar{\psi}_{\tau}=\tau \bar{\psi}+(1-\tau) a \geq \min (\bar{\psi}, a)>0 .
$$

Applying Lemma 7 to $q_{1}=\Psi$ and $q_{0}=a$, we have

$$
\begin{aligned}
\underline{\lambda}_{1}\left(\Psi_{\tau}\right) & \geq \tau \underline{\lambda}_{1}(\Psi)+(1-\tau) \underline{\lambda}_{1}(a) \\
& \geq \min \left(\underline{\lambda}_{1}(\Psi), \underline{\lambda}_{1}(a)\right)>0 .
\end{aligned}
$$

Thus $\psi_{\tau}$ and $\Psi_{\tau}$ defined above satisfy (17) uniformly in $\tau \in$ $[0,1]$.

Lemma 8. For every $\Gamma>0$, there exists a constant $\mu(\Gamma) \geq 1$ such that if $\mu \geq \mu(\Gamma), \tau \in[0,1]$, and $\rho$ is a T-periodic solution of (36), then $\|\rho\| \geq \Gamma$.

Proof. Assume by contradiction that there exist $\Gamma>0$ and sequence $\left\{\tau_{n}\right\},\left\{\mu_{n}\right\}$, and $\left\{\rho_{n}\right\}$ such that $\left\{\tau_{n}\right\} \subset[0,1]$, $\lim _{n \rightarrow \infty} \mu_{n}=+\infty$, and $\rho_{n}$ is a $T$-periodic solution of (36) for $\tau=\tau_{n}$ and $\mu=\mu_{n}$ with $\left\|\rho_{n}\right\|<\Gamma$.

From $\left(\mathrm{H}_{1}\right)$, there exist $c^{\prime \prime}>0$ and $\delta^{\prime}>0$ such that

$$
f(t, s ; \tau) \leq-\frac{c^{\prime \prime}}{s^{\nu}}<0 \quad \text { when } 0<s \leq \delta^{\prime},
$$

and from $\left(\mathrm{H}_{2}\right)$,

$$
\limsup _{\rho \rightarrow+\infty} \frac{f(t, \rho ; \tau)}{\rho} \leq \Psi(t)<\max _{t} \Psi(t)
$$

uniformly in $\tau \in[0,1]$; one knows that there are some constants $M, N$ such that

$$
f(t, s ; \tau)<M s+N \quad \forall s>0 \text { and all } t .
$$

Multiplying (36) by $\rho_{n}^{3}(t)$ and integrating from 0 to $T$, we obtain

$$
\begin{aligned}
& -3 \int_{0}^{T} \dot{\rho}_{n}^{2}(t) \rho_{n}^{2}(t) d t+\int_{0}^{T} f\left(t, \rho_{n}(t) ; \tau\right) \rho_{n}^{3}(t) d t \\
& =\mu_{n}^{2} T .
\end{aligned}
$$

Therefore,

$$
\mu_{n}^{2} T<\int_{0}^{T} f\left(t, \rho_{n}(t) ; \tau\right) \rho_{n}^{3}(t) d t \leq \frac{M T \Gamma^{5}}{5}+\frac{N T \Gamma^{4}}{4},
$$

which is a contradiction with the fact that $\mu_{n} \rightarrow+\infty$.
Lemma 9. Fix $\bar{\mu}=\mu(\Gamma)$ as in Lemma 8. Given $A, B$ with $\bar{\mu} \leq$ $A \leq B$, there exists $C_{2}>C_{1}>0$ such that if $\mu \in[A, B]$ and $\rho(t)$ is a positive T-periodic solution of (36), then

$$
C_{1}<\rho\left(t_{0}\right)<C_{2},
$$

for some $t_{0} \in[0, T]$.

Proof. Let $\rho(t)$ be a positive $T$-periodic solution of (36). From $\left(\mathrm{H}_{1}\right)$, we know that there exist positive constants $c_{1}, c_{1}^{\prime}, \delta_{1}, \nu_{1}=\max \{\nu, 3\} \geq 1$ such that

$$
\frac{c_{1}}{\rho^{\nu_{1}}} \leq-f(t, \rho ; \tau)+\frac{\mu^{2}}{\rho^{3}} \leq \frac{c_{1}^{\prime}}{\rho^{\nu_{1}}}
$$

for all $t$ and $0<\rho \leq \delta_{1}$. Thus, there is $C_{1}>0$ such that

$$
f(t, s ; \tau)-\frac{\mu^{2}}{s^{3}}<-\frac{c_{1}}{s^{\nu_{1}}}<0 \quad \text { when } 0<s<C_{1}:=\delta_{1} .
$$

Integrate (36) from 0 to $T$; we get

$$
\int_{0}^{T} \rho^{\prime \prime}(t) d t+\int_{0}^{T}\left[f(t, \rho(t) ; \tau)-\frac{\mu^{2}}{\rho^{3}(t)}\right] d t=0 .
$$

Thus $\int_{0}^{T}\left[f(t, \rho(t) ; \tau)-\mu^{2} / \rho^{3}(t)\right] d t=0$; there exists $t^{*} \in[0, T]$ such that $\rho\left(t^{*}\right)>C_{1}$.

Take some constant $\varepsilon_{0} \in\left(0, \min \left\{\bar{\psi}, \underline{\lambda}_{1}(\Psi)\right\}\right)$. From $\left(\mathrm{H}_{2}\right)$ there is $C_{2}\left(>C_{1}\right)$ large enough such that

$$
\psi(t)-\varepsilon_{0} \leq \frac{f(t, s ; \tau)}{s}-\frac{\mu^{2}}{s^{4}} \leq \Psi(t)+\varepsilon_{0},
$$

for all $t$ and $s \geq C_{2}$. We assert that $\rho\left(t_{*}\right)<C_{2}$ for some $t_{*}$. Otherwise, assume that $\rho(t) \geq C_{2}$ for all $t$.

Let

$$
\begin{aligned}
h(t) & :=\frac{f(t, \rho(t) ; \tau)}{\rho(t)}-\frac{\mu^{2}}{\rho^{4}(t)} \\
& \in\left(\psi(t)-\varepsilon_{0}, \Psi(t)+\varepsilon_{0}\right) .
\end{aligned}
$$

For $\rho(t) \in L^{1}(0, T)$, let $\bar{\rho}=(1 / T) \int_{0}^{T} \rho(t) d t$; moreover, write $\rho$ as $\rho=\tilde{\rho}+\bar{\rho}$; then $\tilde{\rho}$ satisfies the following differential equation:

$$
\tilde{\rho}^{\prime \prime}+h(t) \tilde{\rho}+h(t) \bar{\rho}=0 .
$$

Integrate (55) from 0 to $T$; we have

$$
\int_{0}^{T} h(t) \tilde{\rho}(t) d t=-\bar{\rho} \int_{0}^{T} h(t) d t .
$$

Multiplying (55) by $\widetilde{\rho}$ and integrating, we get

$$
\begin{aligned}
\left\|\tilde{\rho}^{\prime}\right\|_{2}^{2} & =\int_{0}^{T} h(t) \tilde{\rho}^{2}(t) d t+\bar{\rho} \int_{0}^{T} h(t) \tilde{\rho}(t) d t \\
& =\int_{0}^{T} h(t) \tilde{\rho}^{2}(t) d t-\bar{\rho}^{2}(t) \int_{0}^{T} h(t) d t \\
& \leq \int_{0}^{T} h(t) \tilde{\rho}^{2}(t) d t
\end{aligned}
$$


where the fact that $\int_{0}^{T} h(t) d t>T\left(\bar{\psi}-\varepsilon_{0}\right)>0$. is used.

Note that $\tilde{\rho}\left(t_{0}\right)=0$ for some $t_{0}, \widetilde{\rho}\left(t_{0}+T\right)=0$; thus $\tilde{\rho}(t) \epsilon$ $H_{0}^{1}\left(t_{0}, t_{0}+T\right)$. We assert that $\tilde{\rho} \equiv 0$. On the contrary, assume that $\widetilde{\rho} \not \equiv 0$. Now by (57), the first Dirichlet eigenvalue

$$
\begin{aligned}
& \lambda_{1}^{D}\left(\left.h\right|_{\left[t_{0}, t_{0}+T\right]}\right) \\
& \quad=\inf _{\substack{\varphi \in H_{0}^{1}\left(t_{0}, t_{0}+T\right) \\
\varphi \neq 0}} \frac{\int_{t_{0}}^{t_{0}+T}\left(\varphi^{\prime 2}(t)-h(t) \varphi^{2}(t)\right) d t}{\int_{t_{0}}^{t_{0}+T} \varphi^{2}(t) d t} \leq 0 .
\end{aligned}
$$

So

$$
\underline{\lambda}_{1}(h)=\min \left\{\lambda_{1}^{D}(h)\right\} \leq 0 .
$$

On the other hand, $h(t)<\Psi(t)+\varepsilon_{0}$,

$$
\underline{\lambda}_{1}(h) \geq \underline{\lambda}_{1}\left(\Psi+\varepsilon_{0}\right)=\underline{\lambda}_{1}(\Psi)-\varepsilon_{0}>0 .
$$

This is a contradiction, which shows that $\widetilde{\rho}=0$; thus $\rho=\bar{\rho}$. Now it follows from (56) that $\bar{\rho}=0$ and $\rho \equiv 0$; this contradicts the fact that $\rho$ is a positive solution. We have proven that $\rho\left(t^{*}\right)>C_{1}$ for some $t^{*} \in[0, T]$ and $\rho\left(t_{*}\right)<C_{2}$ for some $t_{*} \in[0, T]$. Thus the intermediate value theorem implies that (49) holds.

Lemma 10. Assume that $\underline{\lambda}_{1}(\Psi)>0$ of the scalar differential equation $x^{\prime \prime}+(\lambda+\Psi(t)) x=0$; then

$$
\begin{aligned}
\left\|x^{\prime}\right\|_{2}^{2} \geq & \int_{0}^{T} \Psi\left(t+t_{0}\right) x^{2}(t) d t \\
& +\lambda_{1}^{D}\left(\Psi_{t_{0}}\right) \int_{0}^{T} x^{2}(t) d t .
\end{aligned}
$$

Proof. By the results for eigenvalues in $\left(\mathrm{E}_{4}\right)$, we have

$$
\lambda_{1}^{D}\left(\Psi_{t_{0}}\right) \geq \underline{\lambda}_{1}(\Psi)>0,
$$

for all $t_{0} \in \mathbb{R}$.

Then, by the theory of linear second-order differential operators [30], the eigenvalues of $x^{\prime \prime}+\left(\lambda+\Psi\left(t+t_{0}\right)\right) x=0$ with Dirichlet boundary conditions form a sequence $\lambda_{1}^{D}\left(\Psi_{t_{0}}\right)<$ $\lambda_{2}^{D}\left(\Psi_{t_{0}}\right)<\cdots$ which tends to $+\infty$, and the corresponding eigenfunctions $\phi_{1}, \phi_{2}, \ldots$ are an orthonormal base of $L^{2}(0, T)$. Hence, given $c_{i} \in \mathbb{R}$ and $x \in H_{0}^{1}(0, T)$, we can write

$$
\begin{aligned}
& x(t)=\sum_{i \geq 1} c_{i} \phi_{i}(t), \\
& \int_{0}^{T}\left(\left(x^{\prime}(t)\right)^{2}-\Psi\left(t+t_{0}\right) x^{2}(t)\right) d t \\
&=\sum_{i \geq 1} c_{i}^{2} \int_{0}^{T}\left(\left(\phi_{i}^{\prime}(t)\right)^{2}-\Psi\left(t+t_{0}\right) \phi_{i}^{2}(t)\right) d t \\
&=\sum_{i \geq 1} c_{i}^{2} \lambda_{i}^{D}\left(\Psi_{t_{0}}\right) \int_{0}^{T} \phi_{i}^{2}(t) d t \\
& \geq \lambda_{1}^{D}\left(\Psi_{t_{0}}\right) \int_{0}^{T} x^{2}(t) d t .
\end{aligned}
$$

This completes the proof.
Lemma 11. Under the assumption in Lemma 9, there exist $C_{3}>C_{2}>0, C_{4}>0$ such that any positive $T$-periodic solution $\rho(t)$ of (36) satisfies

$$
\begin{array}{r}
\|\rho\|<C_{3}, \\
\left\|\rho^{\prime}\right\|<C_{4} .
\end{array}
$$

Proof. Notice the inequality (51). By (16), letting $\varepsilon_{0} \in$ $\left(0, \underline{\lambda}_{1}(\Psi)\right)$, there will be some $\Delta_{1}>\delta_{1}$ such that

$$
f(t, s ; \tau)-\frac{\mu^{2}}{s^{3}} \leq\left(\Psi(t)+\varepsilon_{0}\right) s
$$

for all $s \geq \Delta_{1}$. Hence, one has some $e_{0}>0$ such that

$$
f(t, s ; \tau)-\frac{\mu^{2}}{s^{3}} \leq\left(\Psi(t)+\varepsilon_{0}\right) s+e_{0}
$$

for all $t$ and $s>0$.

Multiplying (36) by $\rho$ and then integrating over $[0, T]$, we get

$$
\begin{aligned}
\left\|\rho^{\prime}\right\|_{2}^{2} & =\int_{0}^{T}\left[f(t, \rho(t) ; \tau)-\frac{\mu^{2}}{\rho^{3}(t)}\right] \rho(t) d t \\
& \leq \int_{0}^{T}\left(\left(\Psi(t)+\varepsilon_{0}\right) \rho(t)+e_{0}\right) \rho(t) d t \\
& =\int_{0}^{T} \Psi(t) \rho^{2}(t) d t+\varepsilon_{0}\|\rho\|_{2}^{2}+e_{0}\|\rho\|_{1} .
\end{aligned}
$$

Note from Lemma 9 that there exists $t_{0}$ which satisfies $C_{1}<\rho\left(t_{0}\right)<C_{2}$. Let $u(t)=\rho\left(t+t_{0}\right)-\rho\left(t_{0}\right)$; then $u \in H_{0}^{1}(0, T)$. Thus

$$
\begin{aligned}
& \int_{0}^{T} \Psi(t) \rho^{2}(t) d t=\int_{0}^{T} \Psi\left(t+t_{0}\right) \rho^{2}\left(t+t_{0}\right) d t \\
& =\int_{0}^{T} \Psi\left(t+t_{0}\right)\left(\rho^{2}\left(t_{0}\right)+2 \rho\left(t_{0}\right) u(t)+u^{2}(t)\right) d t \\
& \leq C_{2}^{2}\|\Psi\|_{1}+2 C_{2}\|\Psi\|_{2}\|u\|_{2} \\
& \quad+\int_{0}^{T} \Psi\left(t+t_{0}\right) u^{2}(t) d t .
\end{aligned}
$$

The other terms in (68) by the Hölder inequality can be estimated as follows:

$$
\begin{aligned}
\varepsilon_{0}\|\rho\|_{2}^{2} & =\varepsilon_{0} \int_{0}^{T}\left(u(t)+\rho\left(t_{0}\right)\right)^{2} d t \\
& \leq \varepsilon_{0}\left(T C_{2}^{2}+2 C_{2} T^{1 / 2}\|u\|_{2}+\|u\|_{2}^{2}\right), \\
e_{0}\|\rho\|_{1} & =\varepsilon_{0} \int_{0}^{T}\left(u(t)+\rho\left(t_{0}\right)\right) d t \\
& \leq e_{0}\left(T C_{2}+T^{1 / 2}\|u\|_{2}\right) .
\end{aligned}
$$

Thus (68) reads as

$$
\begin{aligned}
\left\|u^{\prime}\right\|_{2}^{2} \leq & A_{0}+B_{0}\|u\|_{2}+\varepsilon_{0}\|u\|_{2}^{2} \\
& +\int_{0}^{T} \Psi\left(t+t_{0}\right) u^{2}(t) d t,
\end{aligned}
$$


where $A_{0}=\varepsilon_{0} T C_{2}^{2}+e_{0} T C_{2}+C_{2}^{2}\|\Psi\|_{1}, B_{0}=2 \varepsilon_{0} C_{2} T^{1 / 2}+$ $e_{0} T^{1 / 2}+2 C_{2}\|\Psi\|_{2}$ are positive constants.

On the other hand, using Lemma 10,

$$
\begin{aligned}
\underline{\lambda}_{1}(\Psi(t))\|u\|_{2}^{2} & \leq \lambda_{1}^{D}\left(\Psi_{t_{0}}\right)\|u\|_{2}^{2} \\
& \leq \int_{0}^{T}\left(u^{\prime 2}(t)-\Psi\left(t+t_{0}\right) u^{2}(t)\right) d t,
\end{aligned}
$$

and we get from (71) that

$$
\left(\varepsilon_{0}-\underline{\lambda}_{1}(\Psi(t))\right)\|u\|_{2}^{2}+B_{0}\|u\|_{2}+A_{0} \geq 0 .
$$

Consequently, $\|u\|_{2}<A_{1}$ for some $A_{1}>0$. By (71), one has $\left\|\rho^{\prime}\right\|_{2}=\left\|u^{\prime}\right\|_{2}<A_{2}$ for some $A_{2}>0$. From these, for any $t \in\left[t_{0}, t_{0}+T\right]$,

$$
\begin{aligned}
|\rho(t)| & \leq\left|\rho\left(t_{0}\right)\right|+\left|\int_{t_{0}}^{t} \rho^{\prime}(t) d t\right| \leq C_{2}+T^{1 / 2}\left\|\rho^{\prime}\right\|_{2} \\
& \leq C_{2}+T^{1 / 2} A_{2}:=C_{3} .
\end{aligned}
$$

Thus $\|\rho\|<C_{3}$ is obtained.

In order to prove (65), we write (36) as

$$
-\rho^{\prime \prime}(t)=H(t):=f(t, \rho ; \tau)-\frac{\mu^{2}}{\rho^{3}(t)} .
$$

As $\int_{0}^{T} H(t) d t=0$, thus $\|H(t)\|_{1}=2\left\|H^{+}(t)\right\|_{1}$. Since $\rho(0)=$ $\rho(T)$, there exists $t_{1} \in[0, T]$ such that $\rho^{\prime}\left(t_{1}\right)=0$. Therefore,

$$
\begin{aligned}
\left\|\rho^{\prime}\right\| & =\max _{0 \leq t \leq T}\left|\rho^{\prime}(t)\right|=\max _{0 \leq t \leq T}\left|\int_{t_{1}}^{t} \rho^{\prime \prime}(s) d s\right| \\
& \leq \int_{0}^{T}\left|f(s, \rho(s) ; \tau)-\frac{\mu^{2}}{\rho^{3}(s)}\right| d s \\
& =2 \int_{0}^{T}\left|H^{+}(s)\right| d s \\
& \leq 2 \int_{0}^{T}\left|\left(\Psi^{+}(s)+\varepsilon_{0}\right) \rho(s)+e_{0}\right| d s \\
& \leq 2\left(\left(\left\|\Psi^{+}\right\|_{1}+T \varepsilon_{0}\right) C_{3}+e_{0} T\right):=C_{4},
\end{aligned}
$$

where $\Psi^{+}(t)=\max \{\Psi(t), 0\}, H^{+}(t)=\max \{H(t), 0\}$.

Next, the positive lower estimates for $m=\min _{t \in[0, T]} \rho(t)$ are obtained from the strong force condition $\left(\mathrm{H}_{1}\right)$.

Lemma 12. Under the assumption in Lemma 9, there exists a constant $C_{5} \in\left(0, C_{1}\right)$ such that any positive solution $\rho(t)$ of (36) satisfies

$$
\rho(t)>C_{5} \quad \forall t .
$$

Proof. From (50), we fix some $B_{1} \in\left(0, C_{1}\right)$ such that

$$
f(t, s ; \tau)-\frac{\mu^{2}}{s^{3}}<-C_{4},
$$

for all $t$ and all $0<s \leq B_{1}$. Assume now that

$$
m=\min _{t \in[0, T]} \rho(t)=\rho\left(t_{2}\right)<B_{1} .
$$

By Lemma $9, \max _{t} \rho(t)>C_{1}$. Let $t_{3}>t_{2}$ be the first time instant such that $\rho(t)=B_{1}$. Then, for any $t \in\left[t_{2}, t_{3}\right]$, we have $\rho(t) \leq B_{1}$. Hence, for $t \in\left[t_{2}, t_{3}\right]$,

$$
\rho^{\prime \prime}(t)=-f(t, \rho(t) ; \tau)+\frac{\mu^{2}}{\rho^{3}(t)}>C_{4} \geq 0 .
$$

As $\rho^{\prime}\left(t_{2}\right)=0, \rho^{\prime}(t)>0$ for $t \in\left(t_{2}, t_{3}\right]$. Therefore, the function $\rho:\left[t_{2}, t_{3}\right] \rightarrow \mathbb{R}$ has an inverse, denoted by $\xi$.

Now multiplying (36) by $\rho^{\prime}(t)$ and integrating over $\left[t_{2}, t_{3}\right]$, we get

$$
\begin{aligned}
& \left|\int_{m}^{B_{1}}-f(\xi(\rho), \rho ; \tau) d \rho\right| \\
& \quad=\left|\int_{t_{2}}^{t_{3}}-f(t, \rho(t) ; \tau) \rho^{\prime}(t) d t\right| \\
& \quad=\left|\int_{t_{2}}^{t_{3}}\left[\rho^{\prime \prime}(t)-\frac{\mu^{2}}{\rho^{3}(t)}\right] \rho^{\prime}(t)\right| d t \\
& \quad=\left|\frac{1}{2}\left(\rho^{\prime}\left(t_{3}\right)\right)^{2}+\frac{1}{2}\left(\frac{\mu^{2}}{B_{1}^{2}-m^{2}}\right)\right| \leq B_{2}
\end{aligned}
$$

for some $B_{2}>0$, where the results from Lemma 11 are used. By (50),

$$
\left|\int_{m}^{B_{1}}-f(\xi(\rho), \rho ; \tau) d \rho\right| \geq \int_{m}^{B_{1}} \frac{c_{1}}{\rho^{\nu_{1}}} d \rho \longrightarrow+\infty,
$$

if $m \rightarrow 0^{+}$. Thus we know from (82) that $m>C_{5}$ for some constant $C_{5}>0$.

Let us denote by $C_{T}^{1}$ the set of $T$-periodic $C^{1}$-functions with the usual norm.

Lemma 13. Given $A, B$ with $\bar{\mu} \leq A \leq B$, there is a continuum $\mathscr{C}_{A, B}$ in $[A, B] \times C_{T}^{1}$, connecting $\{A\} \times C_{T}^{1}$ with $\{B\} \times C_{T}^{1}$, whose elements $(\mu, \rho)$ are solutions of equation

$$
\rho^{\prime \prime}+f(t, \rho)-\frac{\mu^{2}}{\rho^{3}}=0 .
$$

Proof. Obviously, if $\mu \geq \bar{\mu}$ and $\rho(t)$ is a $T$-periodic solution of (36), then $\rho(t)$ also satisfies (83). Let us define the following operators:

$$
L: D(L) \subset C^{1}[0, T] \longrightarrow L^{1}(0, T),
$$

$D(L)$

$$
\begin{aligned}
& =\left\{\rho \in W^{2,1}(0, T): \rho(0)=\rho(T), \rho(0)=\rho(T)\right\}, \\
L \rho & =\rho^{\prime \prime}-\rho,
\end{aligned}
$$

$$
N:[A, B] \times C^{1}[0, T] \longrightarrow L^{1}(0, T),
$$$$
N(\mu, \rho)(t)=-f(t, \rho(t))+\frac{\mu^{2}}{\rho^{3}(t)}-\rho(t) .
$$ 
It is clear that (83) is equivalent to the operator equation

$$
L \rho=N(\mu, \rho) \text {. }
$$

Since $L$ is invertible, thus we have

$$
\rho-L^{-1} N(\mu, \rho)=0 .
$$

Define $C=\max \left\{1 / C_{5}, C_{3}, C_{4}\right\}$ and let the open bounded subset in $C^{1}[0, T]$ be

$$
\begin{aligned}
\Omega & =\left\{\rho \in C^{1}[0, T]: \frac{1}{C}<\rho(t)<C,\left|\rho^{\prime}(t)\right|\right. \\
& <C \text { for every } t \in[0, T]\} .
\end{aligned}
$$

By Lemmas 11 and 12, (86) has no solutions $(\mu, \rho)$ on $[A, B] \times$ $\partial \Omega$. Since $L^{-1} N(\mu, \cdot)$ is a compact operator, by Lemma 4 , the result will be proved if we can show that the degree is nonzero for some $\mu \in[A, B]$.

In order to compute the degree, we consider (36). By Lemmas 11 and 12, the degree has to be the same for every $\tau \in[0,1]$. Therefore, we consider (36) with $\tau=0$, which is the equation

$$
\rho^{\prime \prime}+a \rho-\frac{\mu^{2}}{\rho^{3}}-\frac{1}{\rho}=0,
$$

which is equivalent to the system

$$
\dot{Y}=F(Y)=\left(u,-a \rho+\frac{\mu^{2}}{\rho^{3}}+\frac{1}{\rho}\right),
$$

where $Y=(\rho, u)$.

It is easy to know that $F$ has a unique zero $\left(\rho_{0}, u_{0}\right)$ and the determinant of Jacobian matrix satisfies $\left|J_{F}\left(\rho_{0}, u_{0}\right)\right|>0$. By Lemma 5, the Leray-Schauder degree of $I-L^{-1} N(\mu, \rho)$ is equal to the Brouwer degree of $F$; that is,

$$
\begin{aligned}
& d_{L}\left(I-L^{-1} N(\mu, \rho), \Omega, 0\right) \\
& \quad=d_{B}\left(F,\left(\frac{1}{C}, C\right) \times(-C, C)\right)=1,
\end{aligned}
$$

and the proof is completed.

We can deduce from Lemma 11 that there is a connected set $\mathscr{C}$, contained in $[\bar{\mu},+\infty] \times C_{T}^{1}$, which connects $\left\{\bar{\mu} \times C_{T}^{1}\right\}$ with $\left\{\mu^{*} \times C_{T}^{1}\right\}$, for every $\mu^{*}>\bar{\mu}$, whose element $(\mu, \rho)$ is the solution of (83).

Lemma 14. For some constants $\bar{\theta}>0$, there exists $\mu_{\theta} \geq \bar{\mu}$ such that if $(\mu, \rho) \in \mathscr{C}$ with $\mu \geq \mu_{\theta}$, then

$$
\int_{0}^{T} \frac{\mu}{\rho^{2}(t)} d t \leq \bar{\theta}
$$

Proof. For $\left(\mathrm{H}_{2}\right)$, there are some $\Delta_{2}>\Delta_{1}$ and small constant $\varepsilon_{1}>0$ such that

$$
f(t, \rho) \leq\left(\Psi(t)+\varepsilon_{1}\right) \rho
$$

for all $\rho(t) \geq \Delta_{2}$. Let $\rho(t)$ be a $T$-periodic solution of (83). Notice inequality (66) and the boundary condition $\int_{0}^{T} \rho^{\prime}(t) d t=0$, by the Wirtinger inequality; we have

$$
\begin{aligned}
(\max \rho-\min \rho)^{2} & \leq T \int_{0}^{T}\left|\rho^{\prime}\right|^{2} d t \leq \frac{T^{3}}{4 \pi^{2}} \int_{0}^{T}\left|\rho^{\prime \prime}\right|^{2} d t \\
& =\frac{T^{3}}{4 \pi^{2}} \int_{0}^{T}\left(f(t, \rho(t))-\frac{\mu^{2}}{\rho^{3}}\right)^{2} d t \\
& \leq \frac{T^{3}}{4 \pi^{2}} \int_{0}^{T}\left(\Psi(t)+\varepsilon_{0}\right)^{2} \rho(t)^{2} d t \\
& =\frac{T^{2}}{4 \pi^{2}}\left(\bar{\Psi}+\varepsilon_{0}\right)^{2}\|\rho\|^{2} .
\end{aligned}
$$

So

$$
\min \rho \geq\left(1-\frac{T}{2 \pi}\left(\bar{\Psi}+\varepsilon_{0}\right)\right)\|\rho\|
$$

For $\Gamma:=2 \pi \Delta_{2} /\left(2 \pi-T\left(\bar{\Psi}+\varepsilon_{0}\right)\right)$, let $\mu(\Gamma)$ be as in Lemma 8 . Set $\mu_{\theta}:=\max \{\mu(\Gamma), \bar{\mu}\}$.

Let $(\mu, \rho)$ be an element of $\mathscr{C}$, with $\mu \geq \mu_{\theta}$. By Lemma 8 and (94), $\rho(t) \geq \Delta_{2}$, for every $t \in \mathbb{R}$. Integrating (83) from 0 to $T$, we have

$$
\begin{aligned}
\frac{1}{T} \int_{0}^{T} \frac{\mu^{2}}{\rho^{3}(t)} d t & =\frac{1}{T} \int_{0}^{T} f(t, \rho(t)) d t \\
& \leq \frac{1}{T} \int_{0}^{T}\left(\Psi(t)+\varepsilon_{1}\right) \rho d t \\
& \leq\left(\bar{\Psi}+\varepsilon_{1}\right)\|\rho\| .
\end{aligned}
$$

Therefore, we obtain

$$
\begin{aligned}
\int_{0}^{T} \frac{\mu}{\rho^{2}(t)} d t & \leq\left(\int_{0}^{T} \frac{\mu^{2}}{\rho^{4}(t)} d t\right)^{1 / 2}\left(\int_{0}^{T} 1^{2} d t\right)^{1 / 2} \\
& =\sqrt{T}\left(\int_{0}^{T} \frac{\mu^{2}}{\rho^{3}(t)} \cdot \frac{1}{\rho(t)} d t\right)^{1 / 2} \\
& \leq \sqrt{\frac{2 \pi T^{2}\left(\bar{\Psi}+\varepsilon_{1}\right)}{2 \pi-T\left(\bar{\Psi}+\varepsilon_{0}\right)}}
\end{aligned}
$$

It follows from the above argument that we can take some constant $\bar{\theta} \in\left(0, \sqrt{2 \pi T^{2}\left(\bar{\Psi}+\varepsilon_{1}\right) /\left(2 \pi-T\left(\bar{\Psi}+\varepsilon_{0}\right)\right)}\right)$, and the proof is finished.

Define the function

$$
\Phi(\mu, \rho) \longmapsto \int_{0}^{T} \frac{\mu}{\rho^{2}(t)} d t .
$$

It is clear that $\Phi$ is continuous from $\mathscr{C}$ to $\mathbb{R}$; Lemma 14 shows that its image is an interval. The following lemma is necessarily the same as Lemma 6 in [1]. The following proof is only for completeness. 
Lemma 15. For every $\theta \in(0, \bar{\theta}]$, there are $(\mu, \rho, \varphi)$, verifying system (32), for which $(\mu, \rho) \in \mathscr{C}$ and

$$
\begin{aligned}
\rho(t+T) & =\rho(t), \\
\varphi(t+T) & =\varphi(t)+\theta,
\end{aligned}
$$

for every $t \in \mathbb{R}$.

Proof. Given $\theta \in(0, \bar{\theta}]$, there are $(\mu, \rho) \in \mathscr{C}$, such that

$$
\Phi(\mu, \rho)=\theta
$$

Obviously, $\rho$ is $T$-periodic satisfying the first equation in (32) and defining

$$
\varphi(t)=\int_{0}^{t} \frac{\mu}{\rho^{2}(s)} d s
$$

it also satisfies the second equation in (32). Moreover

$$
\varphi(t+T)-\varphi(t)=\int_{t}^{t+T} \frac{\mu}{\rho^{2}(s)} d s=\int_{0}^{T} \frac{\mu}{\rho^{2}(s)} d s=\theta
$$

For every $\theta \in(0, \bar{\theta}]$, let $x(t)$ be a solution of system (32). Then it follows from Lemma 15 that

$$
\begin{aligned}
x(t+T) & =\rho(t+T) e^{i \varphi(t+T)}=\rho(t) e^{i \varphi(t)} \cdot e^{i \theta} \\
& =x(t) e^{i \theta} .
\end{aligned}
$$

In particular, if $\theta=2 \pi / k$ for some integer $k \geq 1$, then $x(t)$ is periodic with minimal period $k T$ and rotates exactly once around the origin in the period time $k T$. Hence, for every integer $k \geq 2 \pi / \bar{\theta}$, we have such a $k T$-periodic solution, which we denote by $x_{k}(t)$. Let $\left(\rho_{k}(t), \varphi_{k}(t)\right)$ be its polar coordinates and let $\mu_{k}$ be its angular momentum. By the above construction, $\left(\mu_{k}, \rho_{k}, \varphi_{k}\right)$ satisfy system $(32),\left(\mu_{k}, \rho_{k}\right) \in$ $\mathscr{C}$, and

$$
\int_{0}^{T} \frac{\mu_{k}}{\rho_{k}^{2}(t)} d t=\frac{2 \pi}{k}
$$

Assume $\left(\mu_{k_{j}}\right)$ is a bounded subsequence, with $\left(\mu_{k_{j}}\right) \in[\bar{\mu}, B]$ for some $B$, using Lemma 11 with $\tau=1$; there exists a constant $C_{3}>0$ such that $\left\|\rho_{k_{j}}\right\|<C_{3}$, and hence

$$
\int_{0}^{T} \frac{\mu_{k_{j}}}{\rho_{k_{j}}^{2}(t)} d t>\frac{\bar{\mu} T}{C_{3}^{2}}
$$

for every $j$, in contradiction with (103), so

$$
\lim _{k \rightarrow \infty} \min \mu_{k}(t)=+\infty
$$

Moreover, by (105) and (94), with $\tau=1$, we have

$$
\begin{aligned}
\lim _{k \rightarrow \infty}\left\|\rho_{k}\right\| & =+\infty, \\
\lim _{k \rightarrow \infty}\left(\min \rho_{k}\right) & =+\infty .
\end{aligned}
$$

The proof of Theorem 6 is finished.

\section{Periodic Solutions with a Small Angular Momentum}

In this section, we establish the periodic orbits of (1) with a small angular momentum. Since some parts of the proof are in the same line of that of Theorem 6, we will outline the proof with the emphasis on the difference.

Let $\mu=0$; (33) can be written as the $T$-periodic problem

$$
\rho^{\prime \prime}+f(t, \rho)=0 \text {. }
$$

And let $X$ be a Banach space of functions such that $C^{1}([0, T]) \subseteq X \subseteq C([0, T])$, with continuous immersions, and set $X_{*}=\{\rho \in X: \min \rho>0\}$.

Define the following two operators:

$$
\begin{aligned}
& \begin{array}{l}
D\left(L_{0}\right)=\left\{\rho \in W^{2,1}(0, T): \rho(0)=\rho(T), \rho^{\prime}(0)\right. \\
\left.\quad=\rho^{\prime}(T)\right\},
\end{array} \\
& L_{0}: D\left(L_{0}\right) \subset X \longrightarrow L^{1}(0, T), \quad L_{0} \rho=\rho^{\prime \prime}, \\
& N_{0}: X_{*} \longrightarrow L^{1}(0, T), \\
& \left(N_{0} \rho\right)(t)=-f(t, \rho(t)) .
\end{aligned}
$$

Taking $\sigma \in \mathbb{R}$ not belonging to the spectrum of $L$, (107) can be translated to the fixed problem

$$
\rho=\left(L_{0}-\sigma I\right)^{-1}\left(N_{0}-\sigma I\right) \rho .
$$

We will say that a set $\Omega \subseteq X$ is uniformly positively bounded below if there is a constant $\delta>0$ such that $\min \rho \geq \delta$ for every $\rho \in \Omega$. In order to prove the main result of this paper, we need the following theorem, which has been proved in [4].

Lemma 16. Let $\Omega$ be an open bounded subset of $X$, uniformly positively bounded below. Assume that there is no solution of (107), on the boundary $\partial \Omega$, and that

$$
\operatorname{deg}\left(I-\left(L_{0}-\sigma I\right)^{-1}\left(N_{0}-\sigma I\right), \Omega, 0\right) \neq 0
$$

Then, there exists a $k_{3} \geq 1$ such that, for every integer $k \geq k_{3}$, system (1) has a periodic solution $x_{k}(t)$ with minimal period $k T$, which makes exactly one revolution around the origin in the period time $k T$. The function $\left|x_{k}(t)\right|$ is $T$-periodic and when restricted to $[0, T]$, it belongs to $\Omega$. Moreover, if $\mu_{k}$ denotes the angular momentum associated with $x_{k}(t)$, then

$$
\lim _{k \rightarrow \infty} \mu_{k}=0
$$

The main result of this section reads as follows.

Theorem 17. Assume that $\left(H_{1}\right)$ and $\left(H_{2}\right)$ are satisfied. Then there exists $K_{2} \geq 1$ such that, for every integer $k \geq K_{2}$, system (1) has a periodic solution $x_{k}(t)$ with minimal period $k T$, which 
makes exactly one revolution around the origin in the period time $k T$. Moreover, there exists a constant $\widetilde{C}>0$ such that

$$
\frac{1}{\widetilde{C}}<\left|x_{k}(t)\right|<\widetilde{C},
$$

$$
\text { for every } t \in \mathbb{R} \text { and every } k \geq K_{2} \text {, }
$$

$$
\lim _{k \rightarrow \infty} \mu_{k}=0
$$

Proof. We consider the T-periodic problem (107). Using the same technique in Section 3, we can prove that there exists a constant $\widetilde{C}>0$ such that if $\rho$ is a $T$-periodic solution of (107), then

$$
\begin{aligned}
\frac{1}{\widetilde{C}} & <\rho(t)<\widetilde{C}, \\
\left|\rho^{\prime}(t)\right| & <\widetilde{C}
\end{aligned}
$$

for every $t \in[0, T]$. Note that $\widetilde{C}$ is independent of $\mu$.

Define

$$
\begin{aligned}
\widetilde{\Omega} & =\left\{\rho \in C^{1}[0, T]: \frac{1}{\widetilde{C}}<\rho(t)<\widetilde{C},\left|\rho^{\prime}(t)\right|\right. \\
& <\widetilde{C} \text { for every } t \in[0, T]\} .
\end{aligned}
$$

Obviously, $\widetilde{\Omega}$ is an open subset of $C^{1}[0, T]$ and (107) has no solutions on $\partial \Omega$. Again by the homotopy invariance of degree and Lemma 5,

$$
\begin{gathered}
\operatorname{deg}\left(I-(L-\sigma I)^{-1}(N-\sigma I), \Omega, 0\right) \\
=\operatorname{deg}\left(a \rho-\frac{1}{\rho}, \Omega \cap \mathbb{R}, 0\right)=1 .
\end{gathered}
$$

Thus, by Lemma 16, the proof of Theorem 17 is thus completed.

\section{Competing Interests}

The authors declare that they have no competing interests.

\section{Acknowledgments}

This work is supported by the National Natural Science Foundation of China (Grant no. 11461016), the Scientific Research Foundation of Hainan University (kyqd1544), and Hainan Natural Science Foundation (Grants nos. 20167246 and 20161007).

\section{References}

[1] A. Fonda and R. Toader, "Periodic orbits of radially symmetric Keplerian-like systems: a topological degree approach," Journal of Differential Equations, vol. 244, no. 12, pp. 3235-3264, 2008.

[2] A. Fonda and R. Toader, "Radially symmetric systems with a singularity and asymptotically linear growth," Nonlinear Analysis: Theory, Methods \& Applications, vol. 74, no. 7, pp. 2485-2496, 2011.
[3] A. Fonda and R. Toader, "Periodic orbits of radially symmetric systems with a singularity: the repulsive case," Advanced Nonlinear Studies, vol. 11, no. 4, pp. 853-874, 2011.

[4] A. Fonda and R. Toader, "Periodic solutions of radially symmetric perturbations of Newtonian systems," Proceedings of the American Mathematical Society, vol. 140, no. 4, pp. 1331-1341, 2012.

[5] A. Fonda, R. Toader, and F. Zanolin, "Periodic solutions of singular radially symmetric systems with superlinear growth," Annali di Matematica Pura ed Applicata, vol. 191, no. 2, pp. 181204, 2012.

[6] A. Fonda and A. J. Ureña, "Periodic, subharmonic, and quasiperiodic oscillations under the action of a central force," Discrete and Continuous Dynamical Systems A, vol. 29, no. 1, pp. 169-192, 2011.

[7] A. C. Lazer and S. Solimini, "On periodic solutions of nonlinear differential equations with singularities," Proceedings of the American Mathematical Society, vol. 99, no. 1, pp. 109-114, 1987.

[8] D. Jiang, J. Chu, and M. Zhang, "Multiplicity of positive periodic solutions to superlinear repulsive singular equations," Journal of Differential Equations, vol. 211, no. 2, pp. 282-302, 2005.

[9] S. Li, F. Liao, and H. Zhu, "Multiplicity of positive solutions to second-order singular differential equations with a parameter," Boundary Value Problems, vol. 2014, article 115, 2014.

[10] S. Li, F. Liao, and W. Xing, "Periodic solutions of Liénard differential equations with singularity," Electronic Journal of Differential Equations, vol. 151, pp. 1-12, 2015.

[11] S. Li, F. Liao, and H. Zhu, "Periodic solutions of second order non-autonomous differential systems," Fixed Point Theory, vol. 15, no. 2, pp. 487-494, 2014.

[12] F. Wang and Y. Cui, "On the existence of solutions for singular boundary value problem of third-order differential equations," Mathematica Slovaca, vol. 60, no. 4, pp. 485-494, 2010.

[13] F. Wang, F. Zhang, and Y. Yu, "Existence of positive solutions of Neumann boundary value problem via a convex functional compression-expansion fixed point theorem," Fixed Point Theory, vol. 11, no. 2, pp. 395-400, 2010.

[14] H. Wang, "On the number of positive solutions of nonlinear systems," Journal of Mathematical Analysis and Applications, vol. 281, no. 1, pp. 287-306, 2003.

[15] H. Wang, "Positive periodic solutions of singular systems with a parameter," Journal of Differential Equations, vol. 249, no. 12, pp. 2986-3002, 2010.

[16] Y. Wang and Y.-H. Xia, "The existence of almost periodic solutions of a certain nonlinear system," Communications in Nonlinear Science and Numerical Simulation, vol. 16, no. 2, pp. 1060-1072, 2011.

[17] W. B. Gordon, "Conservative dynamical systems involving strong forces," Transactions of the American Mathematical Society, vol. 204, pp. 113-135, 1975.

[18] P. Majer and S. Terracini, "Periodic solutions to some problems of n-body type," Archive for Rational Mechanics and Analysis, vol. 124, no. 4, pp. 381-404, 1993.

[19] S. Zhang, "Multiple closed orbits of fixed energy for $N$ body-type problems with gravitational potentials," Journal of Mathematical Analysis and Applications, vol. 208, no. 2, pp. 462475, 1997.

[20] A. Fonda, R. F. Manásevich, and F. Zanolin, "Subharmonic solutions for some second-order differential equations with singularities," SIAM Journal on Mathematical Analysis, vol. 24, no. 5, pp. 1294-1311, 1993. 
[21] M. A. del Pino and R. F. Manásevich, "Infinitely many Tperiodic solutions for a problem arising in nonlinear elasticity," Journal of Differential Equations, vol. 103, no. 2, pp. 260-277, 1993.

[22] M. A. del Pino, R. F. Manásevich, and A. Montero, “T-periodic solutions for some second order differential equations with singularities," Proceedings of the Royal Society of Edinburgh A, vol. 120, no. 3-4, pp. 231-243, 1992.

[23] P. Yan and M. Zhang, "Higher order non-resonance for differential equations with singularities," Mathematical Methods in the Applied Sciences, vol. 26, no. 12, pp. 1067-1074, 2003.

[24] M. Zhang, "A relationship between the periodic and the Dirichlet BVPs of singular differential equations," Proceedings of the Royal Society of Edinburgh. Section A. Mathematics, vol. 128, no. 5, pp. 1099-1114, 1998.

[25] W. Magnus and S. Winkler, Hill's Equation, Dover, New York, NY, USA, 1979.

[26] M. Zhang, "The rotation number approach to eigenvalues of the one-dimensional p-Laplacian with periodic potentials," Journal of the London Mathematical Society, vol. 64, no. 1, pp. 125-143, 2001.

[27] E. Zeidler, Nonlinear Functional Analysis and Its Applications, vol. 1, Springer, New York, NY, USA, 1986.

[28] A. Capietto, J. Mawhin, and F. Zanolin, "Continuation theorems for periodic perturbations of autonomous systems," Transactions of the American Mathematical Society, vol. 329, no. 1, pp. 41-72, 1992.

[29] J. Mawhin, "Topological degree methods in nonlinear boundary value problems," CBMS Regional Conference Series in Mathematics, vol. 40, 122 pages, 1979.

[30] W. N. Everitt, M. K. Kwong, and A. Zettl, "Oscillation of eigenfunctions of weighted regular Sturm-Liouville problems," Journal of the London Mathematical Society, vol. 27, no. 1, pp. 106-120, 1983. 


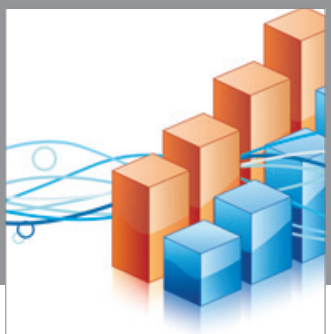

Advances in

Operations Research

vatem alat4

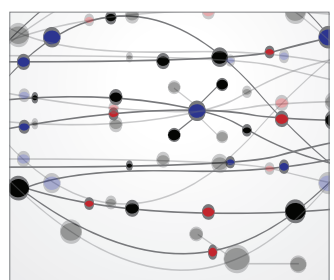

\section{The Scientific} World Journal
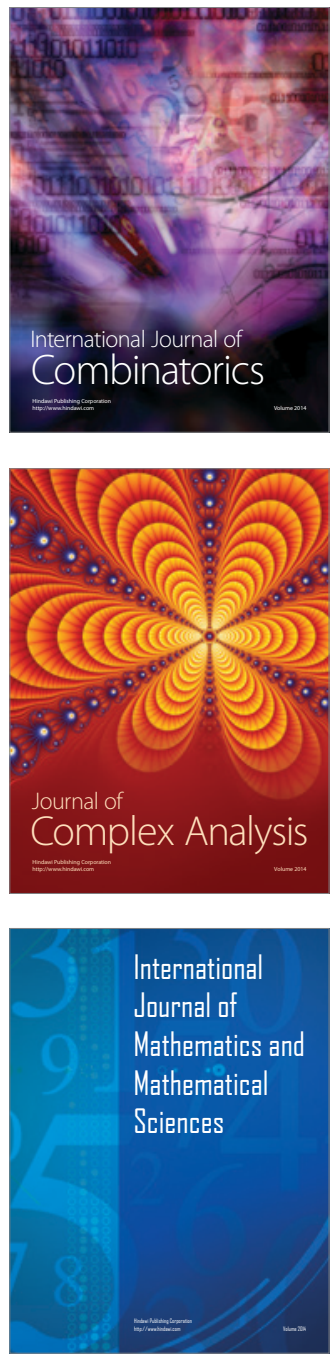
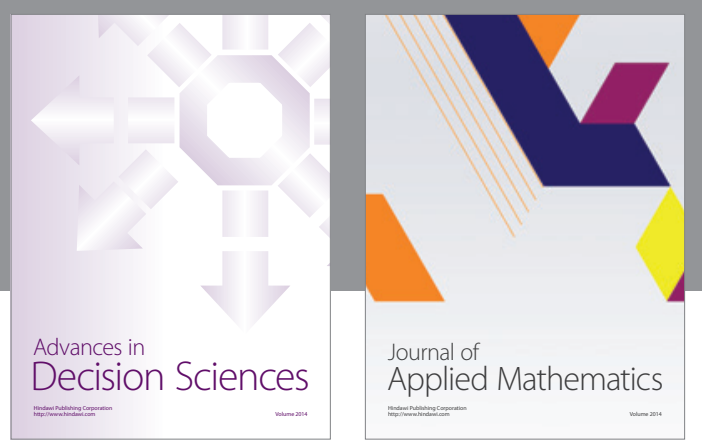

Algebra

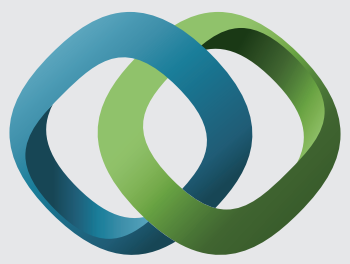

\section{Hindawi}

Submit your manuscripts at

http://www.hindawi.com
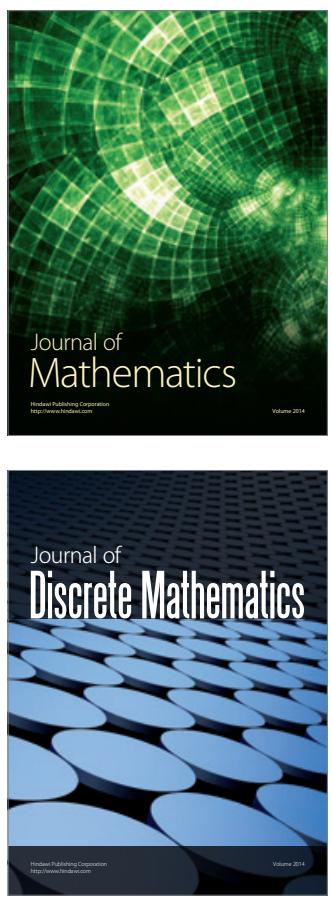

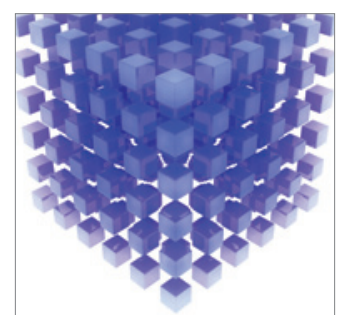

Mathematical Problems in Engineering
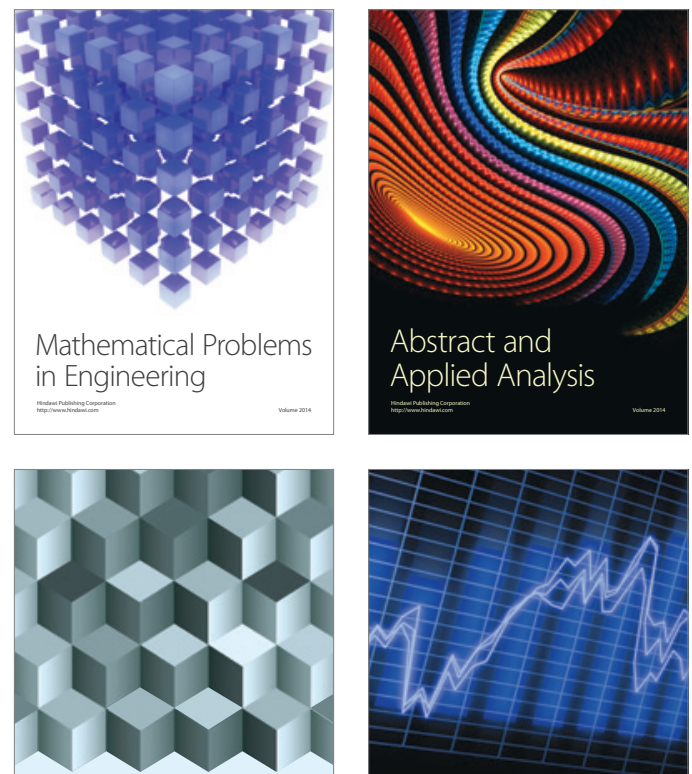

Journal of

Function Spaces

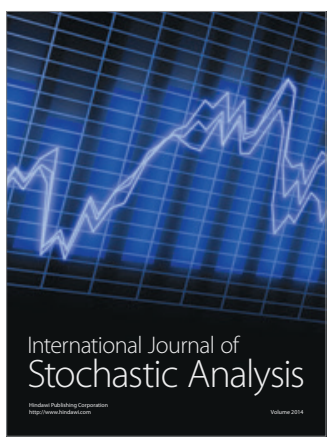

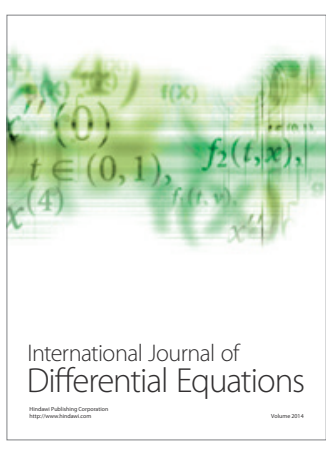
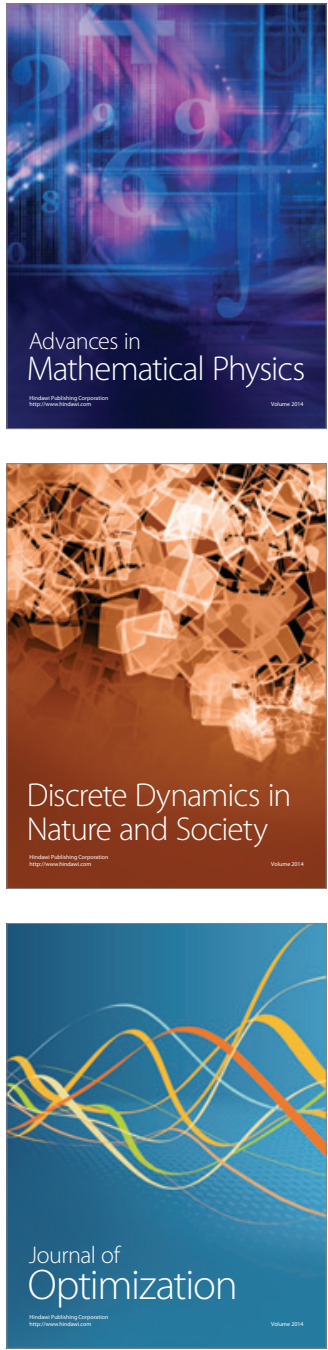